\section{Weniger Genitalwarzen durch den bivalenten HPV-16/18-Impfstoff}

Der bivalente HPV-16/18-Impfstoff zeigte in klinischen Studien ein hohe Wirksamkeit in der Prävention von höhergradigen zervikalen intraepithelialen Neoplasien, verursacht durch die onkogenen HPV-16, 18 sowie aufgrund einer Kreuzprotektion auch gegen HPV-31, 33 und 45. Seit Einführung des HPVImpfprogramms im Vereinigten Königreich 2008 verringerten sich unter dieser HPV-Vakzine auch Genitalwarzen. Wie lässt sich diese Wirkung erklären?

Seit den 1970er-Jahren ist in England die $S_{\text {jährliche Fallzahl an Genitalwarzen, }}$ die in genitourologischen Ambulanzen (GUM) diagnostiziert wurden, stetig gestiegen (Ausnahme: leichter Rückgang 1987-1995 im Rahmen der AIDS-Aufklärung). 2009 wurde erstmals ein deutlicher Rückgang von 37.062 Fällen im Jahr 2008 auf 35.925 beobachtet. Auch 2010 und 2011 wurden weniger als 35.000 Erkrankungsfälle registriert. Dieser Rückgang fand sich nur bei Genitalwarzen (Mädchen, junge Frauen), nicht aber bei anderen sexuell übertragbaren Erkrankungen. Das Vereinigte Königreich führte 2008 als erstes Land ein kostenfreies nationales Impfprogramm gegen humane Papillomviren (HPV) unter ausschließlicher Verwendung des bivalenten HPV-16/18-Impfstoffs ein: 12-13-jährige Mädchen wurden schulbasiert geimpft. Eine Catch-up-Impfung erfolgte bis zum Alter von 18 Jahren. Das Impfprogramm war mit einer Durchimpfrate von $80 \%$ bei den $12-13$-Jährigen sowie $40 \%$ bei den Mädchen der Catch-
up-Kohorte sehr erfolgreich. Die beiden verfügbaren HPV-Impfstoffe erwiesen sich in klinischen Studien als hochwirksam in der Prävention höhergradiger zervikaler intraepithelialer Neoplasien. Für den tetravalenten HPV-6/11/16/18-Impfstoff konnte in einem nationalen Impfprogramm in Australien zudem eine deutliche Verminderung von Genitalwarzen beobachtet werden. Bei der Einführung des Impfprogramms im Vereinigten Königreich war mit dem bivalenten HPV-16/18Impfstoff kein Rückgang von Genitalwarzen erwartet worden. In einer Studie der Health Protection Agency (England) wurden jetzt die Fallmeldungen von Genitalwarzen aus GUM und hausärztlichen Praxen, das Alter der Patienten und der Impfstatus ausgewertet. Insgesamt betrug der Rückgang von Genitalwarzen in den GUM von 2008-2011 bei 16-19-jährigen Mädchen 13,3\%. Der höchste Rückgang fand sich bei 17 -Jährigen mit $20,8 \%$, bei denen eine Durchimpfrate gegen HPV$16 / 18$ von $64 \%$ angenommen wurde. Der gleiche Trend fand sich in den hausärztlichen Praxen. Die Autoren folgern aus den Ergebnissen, dass die bivalente HPV-16/18 Vakzine auch einen Schutz vor nicht in der Vakzine enthaltenen Condylome-verursachenden HPV-Typen bietet. Als Erklärung führen sie eine Vakzine-induzierte breite Kreuzprotektion an, die offensichtlich auch nicht onkogene Typen einschließt.

Fazit: Dieser unerwartete klinische Effekt der bivalenten HPV-16/18-Vakzine bei der Reduktion von Genitalwarzen lässt sich durch eine B- als T-zelluläre Kreuzprotektion erklären. In einer Post-hoc-Analyse der PATRICIA-Studie, welche die klinische Wirksamkeit gegen HPV-16/18 untersuchte, fand sich eine Wirksamkeit vor sechs Monate persistierenden Infektionen durch die nicht onkogenen HPV-6, 11, 53 und 74 von etwa $34 \%$. Diese Ergebnisse wurden in der gleichen Ausgabe des Journal of Infectious Diseases publiziert und geben somit die wissenschaftliche Erklärung für den von den englischen Gesundheitsbehörden berichteten Rückgang von Genitalwarzen. Für nationale Impfprogramme bedeutet dies, bedingt durch die Kreuzprotektion gegen HPV-31, 33 und 45 , nicht nur eine breite Wirksamkeit gegen onkogene HPVs, sondern auch einen gewissen Zusatzeffekt durch die Reduktion von Genitalwarzen. Prof. Tino F.Schwarz

Howell-Jones R et al.: Declining genital warts in young women in England associated with HPV16/18 vaccination: an ecological study. J Infect Dis (accepted)

\title{
Metformin hilft beim Ovarialkarzinom
}

Es gibt Hinweise, dass Metformin eine antikarzinogene Wirkung besitzt. Die Autoren fanden eine derartige Wirkung von Metformin auch in experimentellen Studien beim Ovarialkarzinom.

A $\mathrm{n}$ einer Fall-Kontroll-Studie nahmen Frauen mit Ovarialkarzinom teil, denen mindestens ein Jahr Metformin in einer Dosierung zwischen 500 und $2000 \mathrm{mg}$ täglich verordnet worden war. Als Kontrollen dienten Patientinnen mit Ovarialkarzinom, die kein Metformin erhalten hatten (nicht diabetische Kontrollen) und jene Frauen, die mit anderen Antidiabetika oder Insulin, nicht aber mit Metformin behandelt wurden (diabetische Kontrollen).

Diabetiker ohne Metforminbehandlung hatten - bei allerdings nur kleinen Fallzahlen - die niedrigste Lebenserwartung (40\%) im Vergleich zu nicht-diabetischen Kontrollen (47\%) und den Patientinnen mit Metformin (67\%).

Kumar S, Meuter A, Thapa P. et al.

Metformin intake is associated with better survival in ovarian cancer. Cancer 2013; 119: 555562
Kommentar: Anlass der Studie waren experimentelle, epidemiologische und klinische Hinweise, nach denen Metformin bei verschiedenen Malignomen eine günstige Wirkung hat. Insofern kommt das Ergebnis nicht überraschend. Wahrscheinlich wird Metformin aber kein Bestandteil der Chemotherapie maligner Tumoren. Die Hoffnungen stützen sich darauf, dass jetzt die antitumoröse Zweitwirkung der Substanz durch Molekülveränderungen herausgefiltert, verstärkt und nutzbar gemacht werden könnte. Der Weg zur Zweitindikationen eines Arzneimittels ist schneller und kostengünstiger als die Entwicklung einer völlig neuen Substanz.

Prof. Heinrich Holzgreve 